\title{
PROBABILISTIC AGENT BASED MODEL FOR TUMORAL CELLS, AND 3D MODEL FOR ANGIOGENESIS
}

\author{
Marta Benítez Aguilar ${ }^{1}$, Luis Saucedo Mora ${ }^{1,2}$, José María Benítez Baena*1, Miguel \\ Ángel Sanz Gómez ${ }^{1}$, Francisco Javier Montáns ${ }^{1,3}$ \\ ${ }^{1}$ Universidad Politécnica de Madrid \\ ETS de ingeniería Aeronautica y del Espacio, Pza Cardenal Cisneros 3, 28040 Madrid \\ 2 Department of Materials, University of Oxford \\ Parks Road, Oxford, OX1 3PJ, UK \\ ${ }^{3}$ Department of Mechanical and Aerospace Engineering, Herbert Wertheim College of Engineering \\ University of Florida, FL 32611, USA
}

Key words: SPH, ABM

\begin{abstract}
.
The study of tumoral cells behaviour through computational models is arising. The movement of these cells is governed by physical laws; therefore, the different forces exerted between them need to be implemented. Nevertheless, biological criteria should be also considered since other factors, as oxygen level or cellular density, are decisive in the real movement. These phenomena are captured by probabilistic models such as the Agent Based Model (ABM). Following this research line, the present paper outlines a numerical model that tries to join both criteria with the aim of reproducing the behaviour of the cells that are part of a brain tumor: Glioblastoma Multiforme (GBM). The study has been carried out by the implementation of the different force equations in a Smoothed-Particle Hydrodynamic (SPH) framework. The SPH method is a meshfree lagrangian method based on the discretization of the study domain into finite particles which carry their own information, as tumoral cells do. Cohesive, viscous and pressure forces have been taking into account. Also, the possible attraction or repulsiveness between cells is considered through the implementation of a mechanical force formulation that combines the Maxwell and KelvinVoigt viscoelastic models. In addition to this forces approach, an energetic model is proposed to consider the results provided by an ABM. It evaluates the energy consumption and the associated extra-force that the cell needs to reach the ABM position, which is considered the biologically optimal one. The model has been tested under different sets of parameters, getting the logical outcome. Successful results have also been found in the evaluation of the energy consumption and, therefore, of the extra-force, finding a formulation that joins both criteria.
\end{abstract}

\section{MECHANICAL MODEL}

The proposed model is based on the Smoothed-Particle Hydrodynamics (SPH) method. The SPH method is a meshfree lagrangian method based on the discretization of the study domain into finite particles, which carry some information of the problem that has to be solved. In the present study, the particles of 

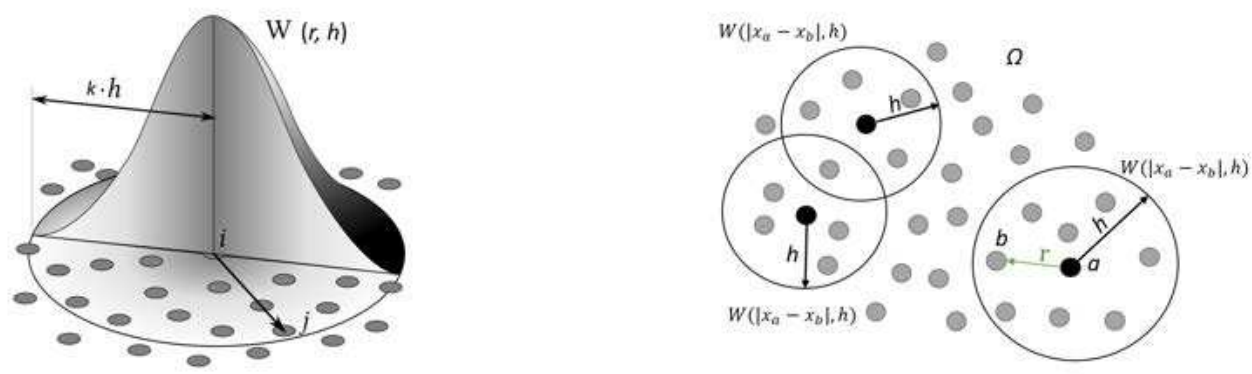

Figure 1: Gaussian kernel function and influence domain

the SPH method represent the discrete domain of cells that are immersed in the tumour tissue.

In order to predict the cells movement, the different forces exerted between each others should be determined. Nevertheless, all of the cells do not interact with all of them, but each one has an influence domain. The length of the influence domain, or radius of influence, is represented by the variable $\mathrm{h}$ and it is determined by the kernel or smoothing function. The cells or particles that are inside it are called neighbours (particles b) and only the neighbourhood of a given particle, a, can modify its movement, exerting forces on it and changing some of its properties, as density or pressure. Every study particle will have a kernel centred on itself and a different radius of influence, $\mathrm{h}$, which will cortespond wi/h the length of its smoothing function. Multiple functions fulfil the required characteristics to [1] (symmetric, positive in the study domain, normalised, etc.) but a study.
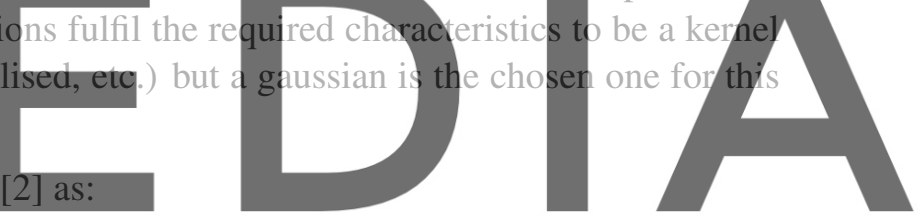

Register for free at https//www.scipedia.dळ

Here, $v$ refers to the number of spatial dimensions and $\sigma$ is a constant that in the present study will take the value of:

$$
\sigma=\frac{h^{3} \rho_{a}}{m_{a}}
$$

To get the value of $h$ all the study domain should be consider. The equation of each $h_{a}$ is:

$$
h_{a}=\frac{r}{\sqrt{\ln (-t o l)}}
$$

where $t o l$ refers to a given tolerance.

And the condition that should be accomplished to get the right value of the distance $r$ (between $a$ and one of each neighbours $b$ ) that appears in the previous equation is: 


$$
\left.\sum_{b=1}^{n} \frac{\mathrm{d} W(r, h)}{\mathrm{d} x_{i}}\right|_{b}=0
$$

Note that $\mathrm{i}=1,2,3$, so there will be three derivatives, one for each spatial dimension.

The main problem of this condition is that the derivative of the kernel function contains the variable $h$, so the equation should be evaluated for each particle $a$ in all of the rest study particles. Due to the meshfree characteristic of the method, the computational cost of this iterative process is affordable. In addition, a previous sifting of the particles that have more likelihood of being neighbours of $a$ could be done. This sifting is done by the creation of a grid with an average value of some $h_{a}$. It creates cubic cells with size $2 h_{\text {average }}$, which enclose the nearest particles, those which are suitable to accomplish the condition imposed by Eq.4. In this study, two grids have been created, one displaced $h$ from the other. These two grids, called odd and even, ensure that every particle is inside one cubic cell. Also, these grids will be helpful to position the particles in the space.

\subsection{General formulation of the SPH method}

Once the neighbours of each particle are determined, the next step will be evaluate the forces between them. Two of the Navier-Stokes equations will be used in the present model: the Mass Conservation and the Momentum Balance. These equations can be rewritten for a discrete domain as follows [3]:

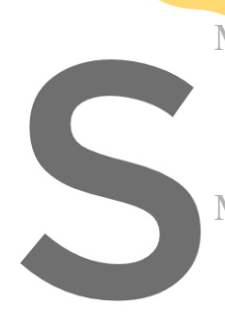

Mass Conservation:

Momentum Balance:
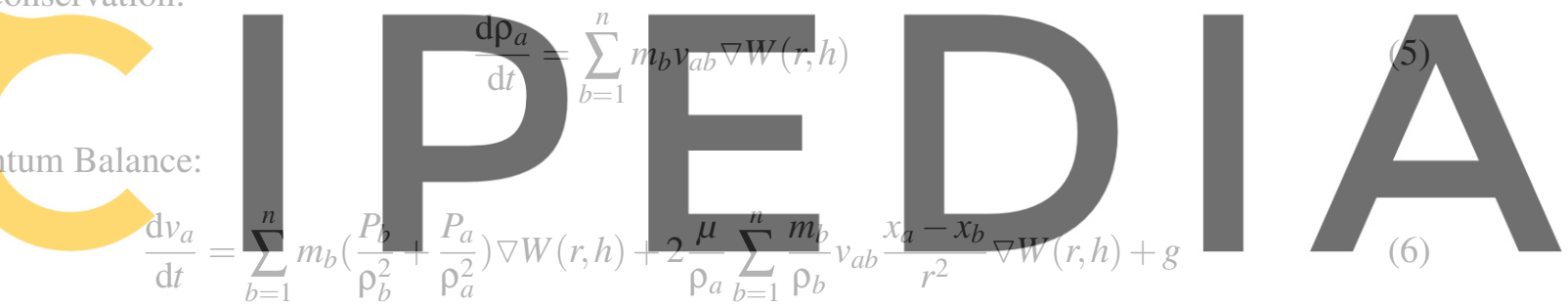

Register for free at https//www.sçipedia.com to download the version without the watermark In the two expressions above $\rho$ is the density, $v$ is the velocity, $t$ refers to time, $P$ is the pressure, $\mu$ is the fluid viscosity $\left(\frac{\mu}{\rho}=v\right.$, the kinematic fluid viscosity $), g$ is the gravity and $v_{a b}=v_{a}-v_{b}$.

Finally, Eq. 5 and Eq. 6 describe the variation of the density and velocity over time, respectively. Nevertheless, it is known that the pressure of the particles will change according to the distances between each other, and that fact has to be captured. For this dynamic case, the fluid can be treated as incompressible and its equation would be:

$$
P=B\left(\left(\frac{\rho}{\rho_{0}}\right)^{\gamma}-1\right)
$$

where $\gamma$ is a constant closer to the value of $7, \rho_{0}$ is the reference density and $B$ is another constant relative to the speed sound, $c$ :

$$
B=\frac{c^{2} \rho_{0}}{\gamma}
$$

The expression 7 was introduced by J. J. Monaghan [4] as another equation of state, part of the three Navier-Stokes equations, and with it, the complete prediction and update of the principal physical properties (density, pressure and acceleration)in a temporal integration method is possible. 


\subsection{Implemented forces}

In the Momentum Balance equation the three summands of the right term, correspond, respectively, to the pressure, viscosity and gravity forces (it should be noted that the forces are all divided by the mass of the particle $a$ in this expression). In the study problem, the gravity is not considered and two more forces have been taking into account: cohesive and mechanical forces.

- Cohesive force: its main parameter is gamma, $\gamma$, and the force rises with it: rising the value of gamma implies rising the attraction between particles.

$$
\frac{F_{\text {cohesive }}}{m_{a}}=\gamma \frac{m_{b}}{m_{a}} W(r, h)\left(x_{a}-x_{b}\right)
$$

- Mechanical force: the pressure force tries to reflect the attractive and repulsive force that appears between cells, but it is not something that could be externally modified to capture any phenomenon. In this case, a viscoelastic model combinig the Kelvin-Voigt and the Maxwell Standard Linear Solid (SLS) models has been chosen to get it. The union of the two models are presented in Figure 2. This viscoelastic model will link each particle with its neighbours, as it is shown in the same figure. Nevertheless, it is important to note that, in the present study, the action of the two represented dashpots is not taken into account, considering only $\eta_{1}$, and the phenomenon that represents the model will be only the storage of energy but not its relaxation effect.
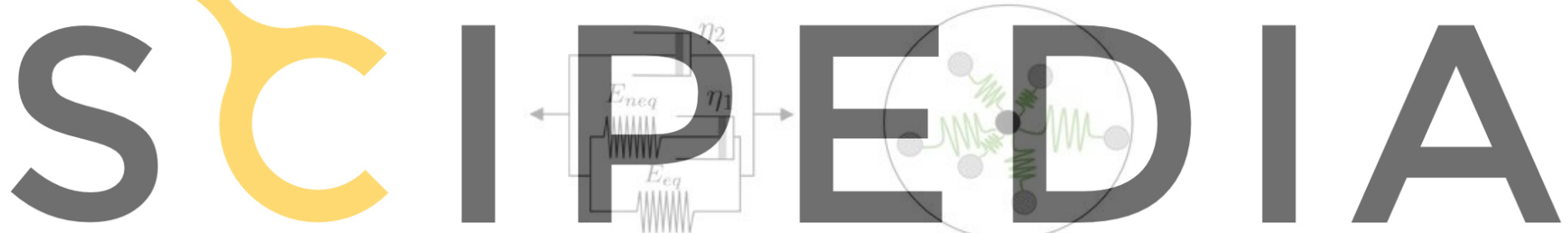

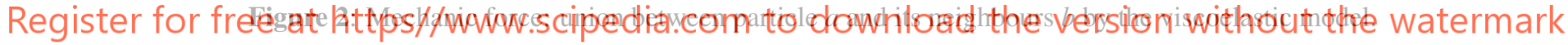

For finite strains and accounting for the viscous behavior, the stress generated by the model could be approximated by:

$$
\sigma=K(1+\hat{\xi}) \frac{2}{\Pi}\left(\lambda_{\text {lim }}-1\right) \tan \left(\frac{\Pi}{2} \frac{\lambda-1}{\lambda_{\text {lim }}-1}\right)
$$

with

$$
\begin{gathered}
\hat{\xi}=\xi \dot{\lambda} \\
\dot{\lambda}=\frac{\lambda^{t}-\lambda^{t-1}}{\Delta t} \\
\lambda=\frac{r_{a b}^{t}}{r_{a b}^{t_{n}}}
\end{gathered}
$$

In the previous expressions $\sigma$ is the stress, $K$ is the string's constant, $\lambda_{\text {lim }}$ is a constant that represents the maximum deformation that the material can develop, $\xi$ is a constant relative to the 
dashpot, $r_{a b}^{t}$ is the distance between $a$ and $b$ in the actual time step, $r_{a b}^{t_{n}}$ is the distance between $a$ and $b$ when they start being neighbours and $\lambda^{t}$ and $\lambda^{t-1}$ refers to Equation 13 particularized for the actual and previous time step, respectively.

Some constants are needed to transform Eq.10 into a force equation. For this model, these constants will be an area of interest, $A$, and a fraction of distances, which is going to give the sign to the force:

$$
\frac{F_{\text {mechanical }}}{m_{a}}=\sigma \frac{A}{m_{a}}\left(\frac{x_{a}-x_{b}}{r_{a b}}\right)
$$

- Viscosity: its main parameter, $\mu$, controls the viscosity of the fluid. When it increases, a higher value of the force is achieved, encouraging the following union of the particles between them and making it difficult to separate. Regarding to its formulation, the viscosity term can be written in terms of the symmetric part of the velocity gradient or rate of deformation tensor, $D$, with the discretization proposed for the SPH method [5]:

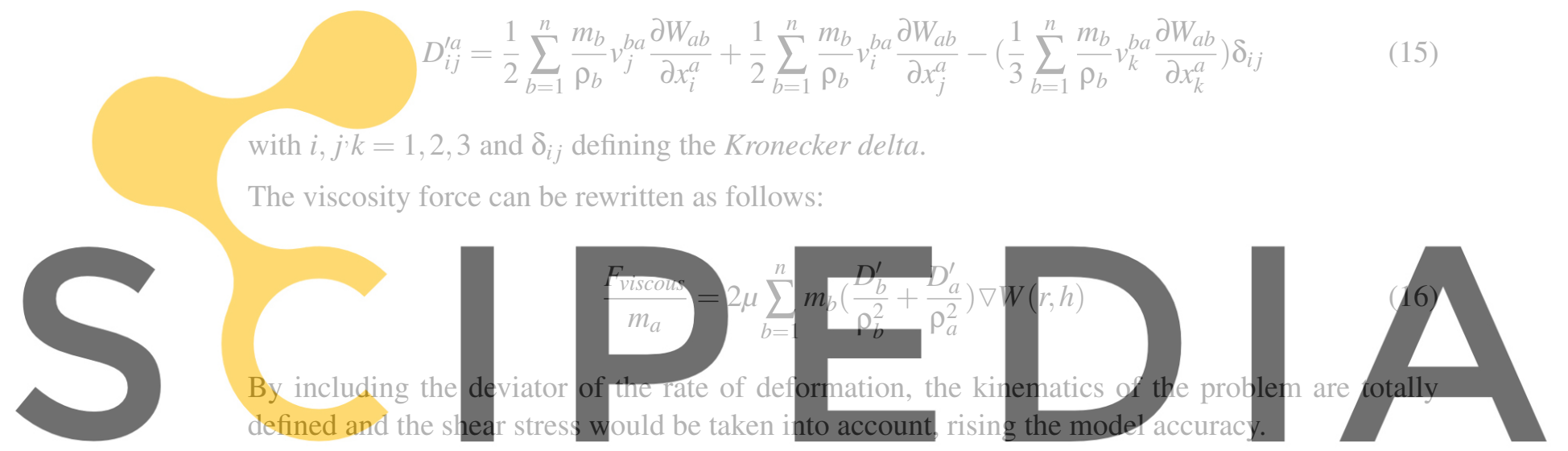

Finally, the complete Momentum Balance equation that has been implemented in the model, including

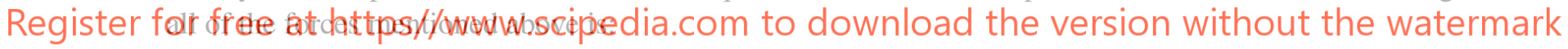

$$
\begin{aligned}
\frac{\mathrm{d} v_{a}}{\mathrm{~d} t}= & \sum_{b=1}^{n} m_{b}\left(\frac{P_{b}}{\rho_{b}^{2}}+\frac{P_{a}}{\rho_{a}^{2}}\right) \nabla W(r, h)+2 \mu \sum_{b=1}^{n} m_{b}\left(\frac{D_{b}^{\prime}}{\rho_{b}^{2}}+\frac{D_{a}^{\prime}}{\rho_{a}^{2}}\right) \nabla W(r, h)+ \\
& +\gamma \frac{m_{b}}{m_{a}} W(r, h)\left(x_{a}-x_{b}\right)+\sum_{b=1}^{n} \frac{\sigma A}{m_{a}}\left(\frac{x_{a}-x_{b}}{r_{a b}}\right)
\end{aligned}
$$

\section{AGENT BASED MODEL}

The mechanical contribution takes the tissue properties and the mechanical phenomena between cells into account. Then, the positions of the particles for the next time step are determined by the Newmar-Beta method, according to the forces exerted on them. To consider the biological criteria, an ABM should be implemented. This model will return other position: the biologically optimal one. In order to reach this new position, the cell will consume as much energy as needed to make a displacement from the position where the forces have carried it, to the mentioned biologically optimal position, as it is represented in 


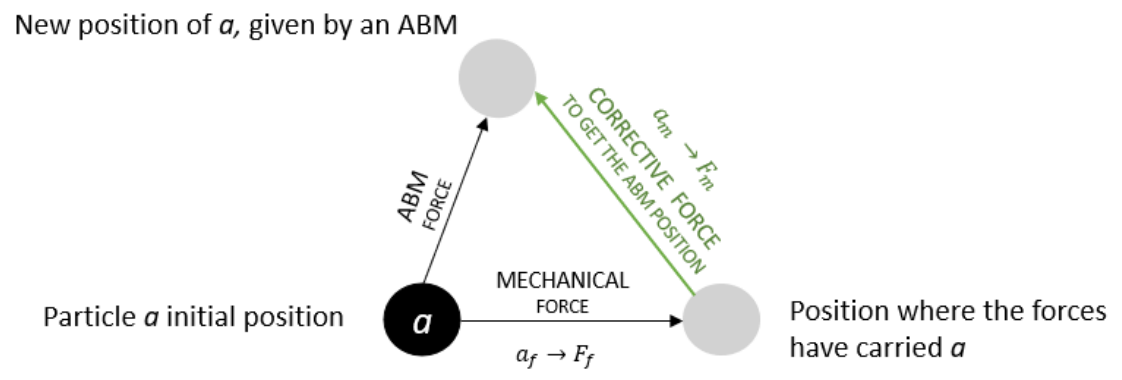

Figure 3: Possible positions of particle $a$

Figure 3. According to the value of this energy consumption, the cell will decide where it will finally move.

The first step to get the real displacement is to calculate the necessary energy consumption to achieve the optimal one. This optimal position will be called ABM position.

The value of the energy consumption needed to go from the force position to the ABM one, is expressed as:
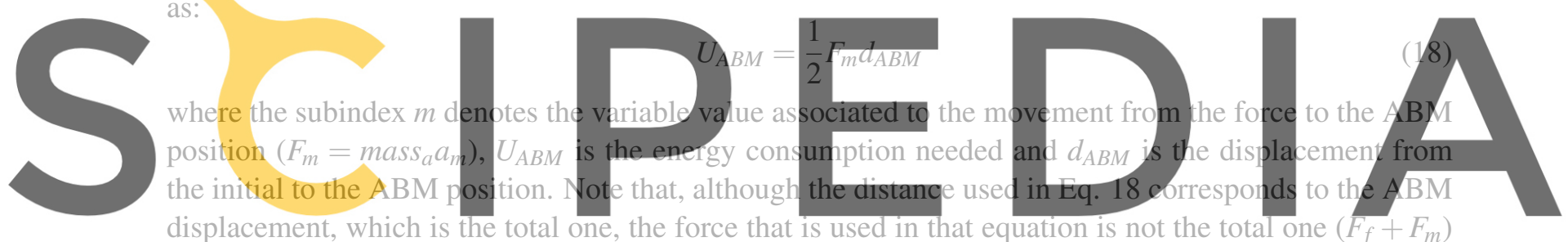

displacement, which is the total one, the force that is used in that equation is not the total one $\left(F_{f}+F_{m}\right)$ because only the $F_{m}$ force leads to an extra-energy cost. Note, also, that the unique unknown variable is

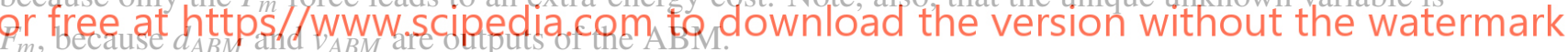

In order to calculate the corrective force that should be exerted in the particle to carry it to the ABM position, $a_{m}$ needs to be determined. To get it, a mathematical development of the Newmark-Beta equations is done by taking the two accelerations presented into account: $a_{t o t}=a_{f}+a_{m}$. With $\gamma=1$ and $\beta=0.5$ it is obtained:

$$
x_{t+1}=\underbrace{x_{t}+\Delta t \dot{x}_{t}+\frac{3 \Delta t^{2}}{2} a_{f}}_{d_{f}}+\underbrace{\frac{3 \Delta t^{2}}{2} a_{m}}_{d_{m}}
$$

and $d_{A B M}=x_{t+1}-x_{t}$, so $a_{m}$ can be easily obtained from the previous expression as:

$$
a_{m}=\left(d_{A B M}-\Delta t \dot{x}_{t}-\frac{3 \Delta t^{2}}{2} a_{f}\right) \frac{2}{3 \Delta t^{2}}
$$

Once the value of $a_{m}$ is known, the value of the energetic cost is also determined by Eq. 18 and the value of the new acceleration is: 


$$
a_{\text {new }}=\frac{F_{f}}{\text { mass }_{a}}+\frac{F_{m}}{\text { mass }_{a}}
$$

This new acceleration can be implemented in the Newmark-Beta method as the real one, expecting to obtain as displacement and velocity for the next step $d_{A B M}$ and $v_{A B M}$, respectively.

\section{RESULTS}

A validation of the whole model should be done before applying it to real problems. Thus, a validation of each part of the model is done in this section, ensuring its proper global functioning.

\subsection{Validation of the searching algorithm of thekernel's length}

To validate the calculations of $h$ and, consequently, the algorithms implemented in Julia for it, a displacement has been imposed without BC particles, inducing a movement of approaching and distancing between cells. This simulation has been carried out without considering the forces to ensure that the SPH framework implementation is correct. The $h$ values are represented by the different colours of the cells. Reddish colours are given for the biggest values of $h$ and they turn into a blue scale when this value decreases. Different times of the simulation are captured in Figure 4.

As it is shown in the mentioned figure, the algorithm asigned higher values of $h$ and, consequently, the particles turn into a red colour, when they are further from each other. On the opposite side, they turn

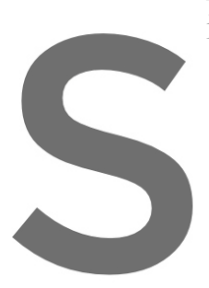
into blue colour when
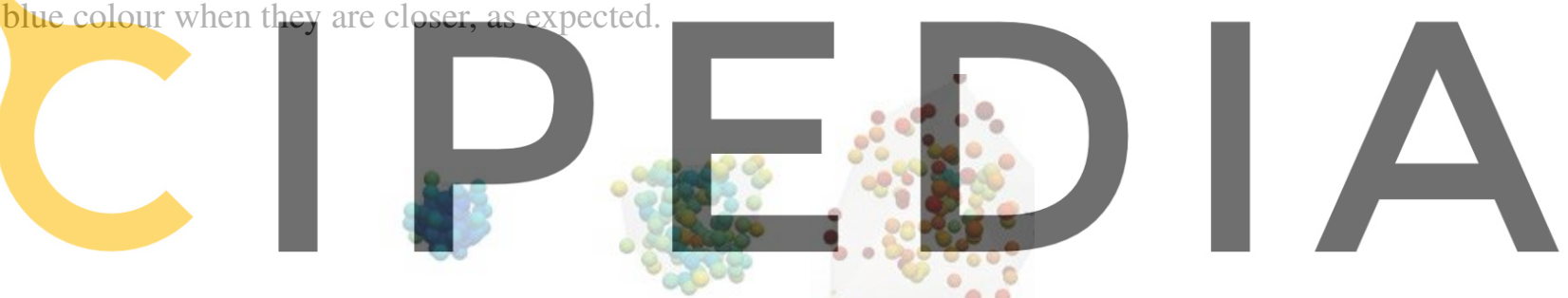

Register for free at https//www.scipedia.com to download the version without the watermark

Figure 4: Validation of $h$

Another way to show the different results of $h$, and validate them, is a representation of the evolution of the different $h$ arithmetic means over time. It can be compared with the theoretical value of $h$ average if the particles were uniformly distributed. Note that the average of the $h$ values is the main datum to obtain the grid which delimits the neighbours searching and, thus, it will be decisive to determine the kindness of the neighbour searching algorithm. This theoretical value is calculated for each time step as follows:

$$
h_{\text {med }}=\frac{1}{2} \sqrt[3]{\frac{V_{\text {total }}}{\text { particles }}}
$$

In Figure 5, the values given by Eq. 22, in which the total volume varies, are represented for each time step (continuous red line); also, the arithmetic mean of the different values of $h$ obtained by the implemented algorithm for each time step is represented by the dotted dark line. The figure shows how 
they are not exactly the same, but their trends are equal and the values obtained by the algorithm are very close to the theoretical ones at all times, proving the kindness of the algorithm.

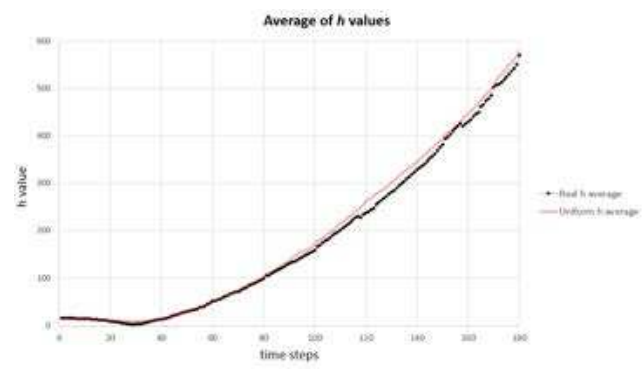

Figure 5: Validation of $h$ : average value of $h$

\subsection{Validation of the implemented forces}

The validation of the forces implementation should also be done. There is not a theoretical or correct value of them. However, their action can be shown in different ways, as graphs over time, varying the main parameters of their equations $(\mu, \gamma$ and $K)$ or by simulating the movement in Paraview and proving the cells act as expected. Note that the value of the forces represented in the graphs is the value of the different forces exerted on a random particle, so its variation through the time its not relevant. Note, also, that the scales of represented magnitude

All the cases have been run in Julia in 2D, with a

$50 \times 50$ square, and withour the square and maintain and will be identified in the different figures w
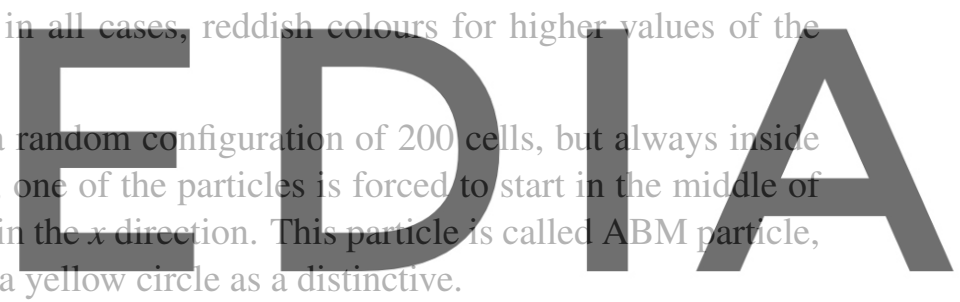

Register for free at https//www.scipedia.com to download the version without the watermark

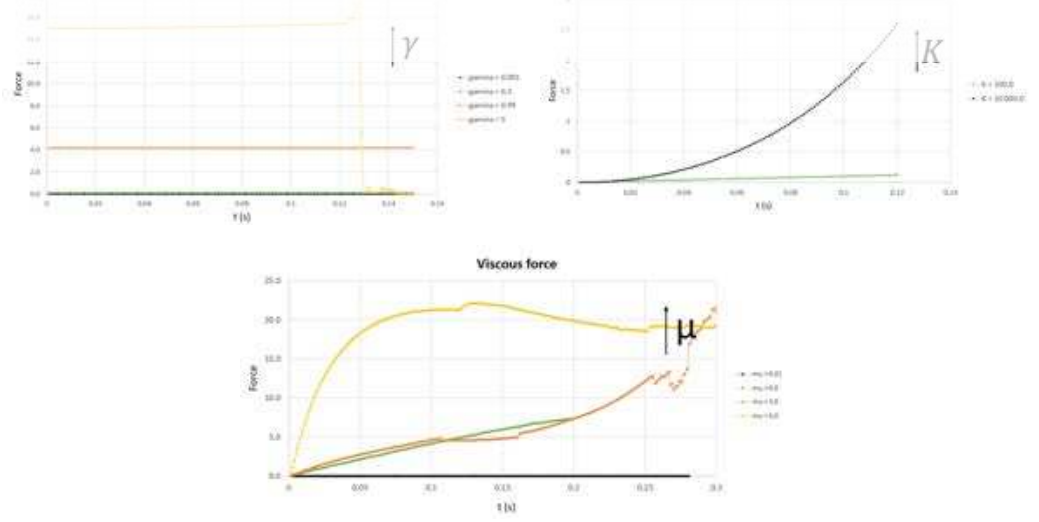

Figure 6: Validation of the forces (cohesive, mechanical and viscous, respectively) by varying their parameters

All of the forces need to rise with its main parameter, as it has been shown in the formulation. The 
Figure 6 shows the different absolute values of each force over the time for different values of their main parameter. For the cohesive force, $\gamma$, will vary between 0.001 and 5.0; for the mechanical force, $K$, will vary between 100.0 and 100000.0 ; and, finally, for the viscous force, $\mu$, will vary between 0.01 and 6.0.

In regards to the results obtained from varying the different parameters that characterize each force, the results are the expected ones. The parameters $\gamma, K$ and $\mu$ are theoretically directly proportional to its force and Figure 6 ensure it. In these graphs, when the main force parameter increases, the value of the force also rises.

In addition, another important fact that should be discussed is the divergence of the forces or the unexpected results in some of the presented cases. This phenomenon appeared in the two first graphs of the Figure 6 for the cohesive and mechanical force, respectively, for the highest value of their parameters. The anomalies presented in the graphs are reflected in the simulation as a big displacement of the cells, that produces an increase in the value of some forces, as the mechanical or the cohesive ones, in an attempt to attract the cell again. This could be the result of a too big $d t$ and a low number of particles choice. Also, these anomalies could be related to the set of parameters chosen. Finding a good set of parameters that gets a balance between these attractive forces and the repulsive ones is needed to get deeper conclusions about the origin of these anomalies.

Concerning the representation of the simulations, Figures 7, 8 and 9 show the behaviour of all the cells according to the different parameters in different time steps.
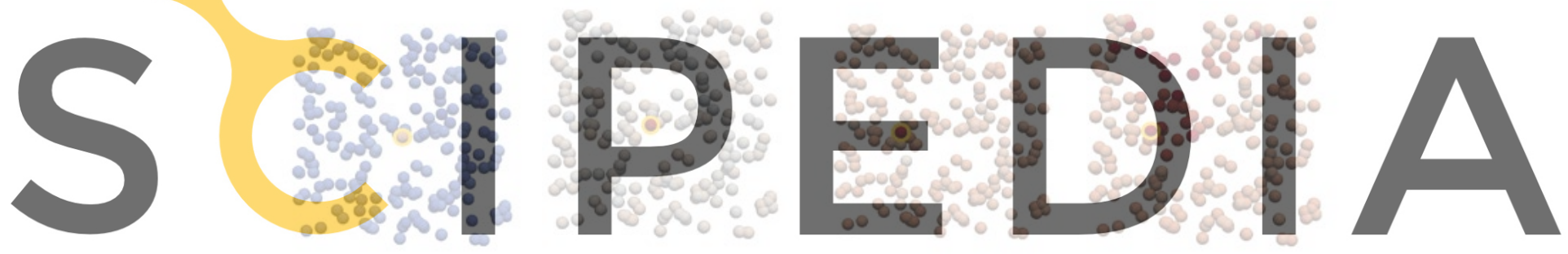

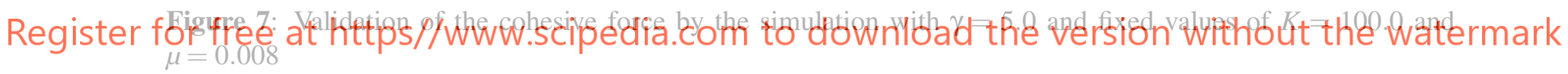
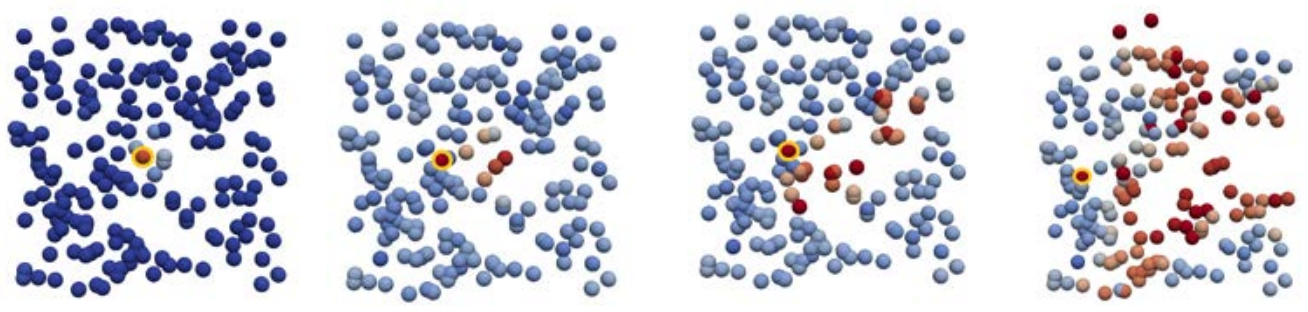

Figure 8: Validation of the mechanical force by the simulation with $K=10000.0$ and fixed values of $\gamma=0.001$ and $\mu=0.008$

In Figure 7, with a high value of $\gamma$ and low of $K$ and $\mu$, the cells start their displacement in a uniform way. The expected result for a high value of $\gamma$ is that all cells move at practically the same time, because this force capture the union between the particles immersed in a fluid. The simulation fulfils the expected 

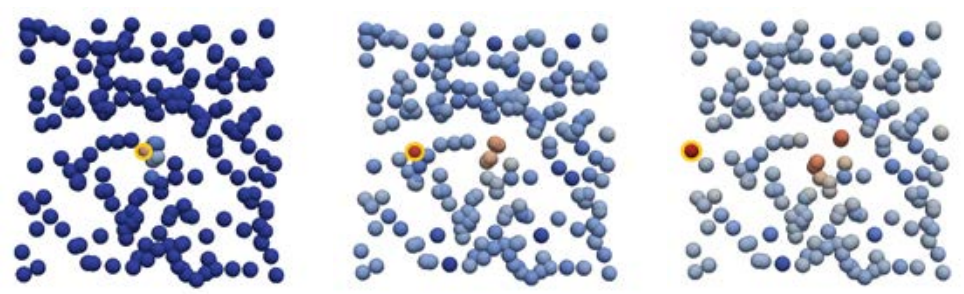

Figure 9: Validation of the viscous force by the simulation with $\mu=6.0$ and fixed values of $\gamma=5.0$ and $K=1000.0$

result and, at the end of the simulation, some of the nearest cells to the ABM one acquire more displacement, due to the action of the mechanical force, which has a medium value. In the case of the Figure $8, \gamma$ and $\mu$ has a lower value and the prevailing force is the mechanical one. In this case, the cells do not move in a uniform way, but only the nearest to the ABM one move, approaching and moving away between each other according to the mechanical force. Finally, for the Figure $9, K$ and $\gamma$ take a higher value, but also $\mu$. In this case, all cells start moving in a uniform way, due to $\gamma$; in a short period of time, the cells nearest the $\mathrm{ABM}$ one start acquiring more displacement, due to the mechanical force; nevertheless, this displacement is, for all particles, lower than in Figure 8, due to the high value of $\gamma$, which tries to maintain the particles linked. Finally, due to the viscous force, the cells nearest the ABM particle start following it. All of these movements are the expected ones according to the physical meaning of each force, which have been exposed in the previous chapter.

As far as pressure force

in Figure 10 and is consiste that the nearest cells of the

the pressure update, Eq.

Thus, until the cells begin

medium, the forces of pres:
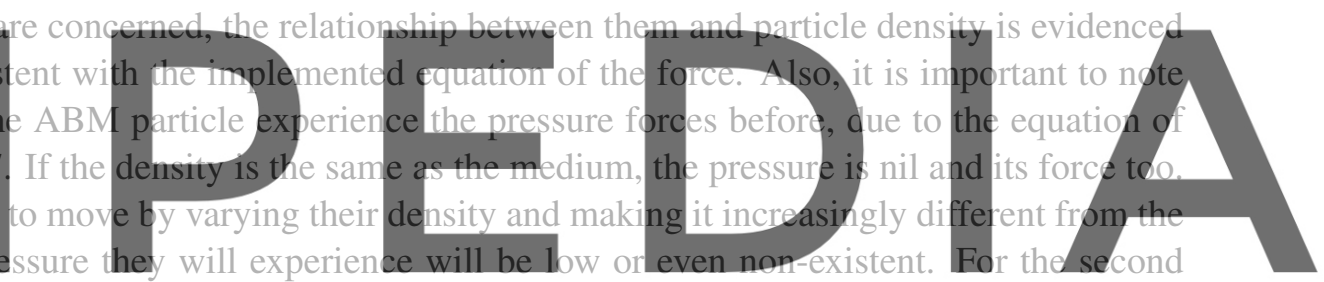

time step, more particles are experimenting pressure forces, because the movement has started to spread.

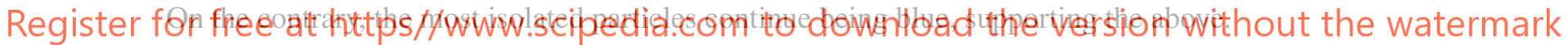

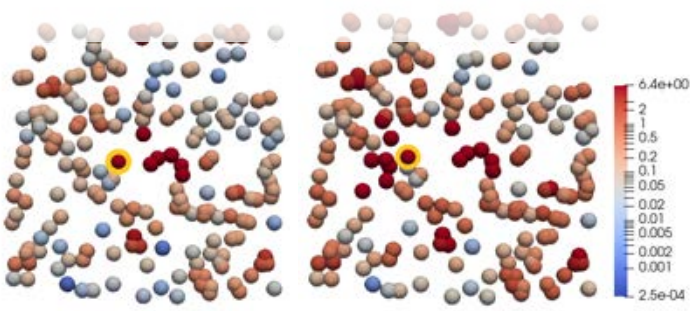

Figure 10: Pressure force exerted on each particle.

\subsection{Validation of the ABM implementation}

Two ways of analysis of the ABM data implementation are proposed: one related to the displacement and velocity continuity over time and other relative to the corrective force. 
For the velocity, first graph in Figure 11, the results given by the NB method are not correct for the first time steps. Nevertheless, the time in which it stabilizes its value is short and this value matches perfectly with the theoretical one. The time that takes the stabilization is acceptable.
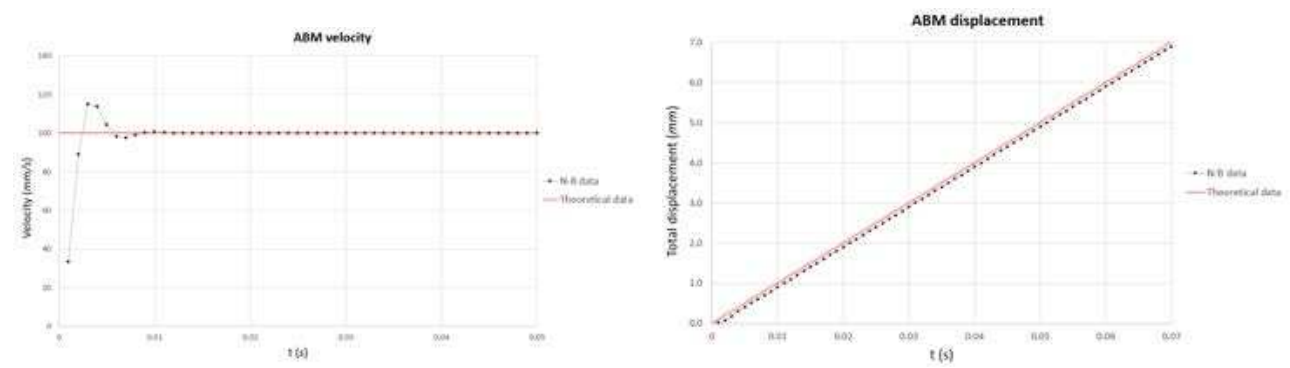

Figure 11: Representation of the ABM velocity and displacement: the expected result (continuous red line) and the result given by Newmark-Beta after the application of the corrective force (dotted dark line)

On the contrary, the displacement, second graph of Figure 11, never stabilizes its value at the theoretical one, getting always a less value. This error persists over time because the displacement is an accumulative parameter. Henceforth, the error in the increment of the displacement, which is the variable returned by the NB method, is only present in the first time steps, as the velocity. If the increment of this variable were not correct for a high time step, the total displacement were not parallel to the theoretical one, but it would take lower values.

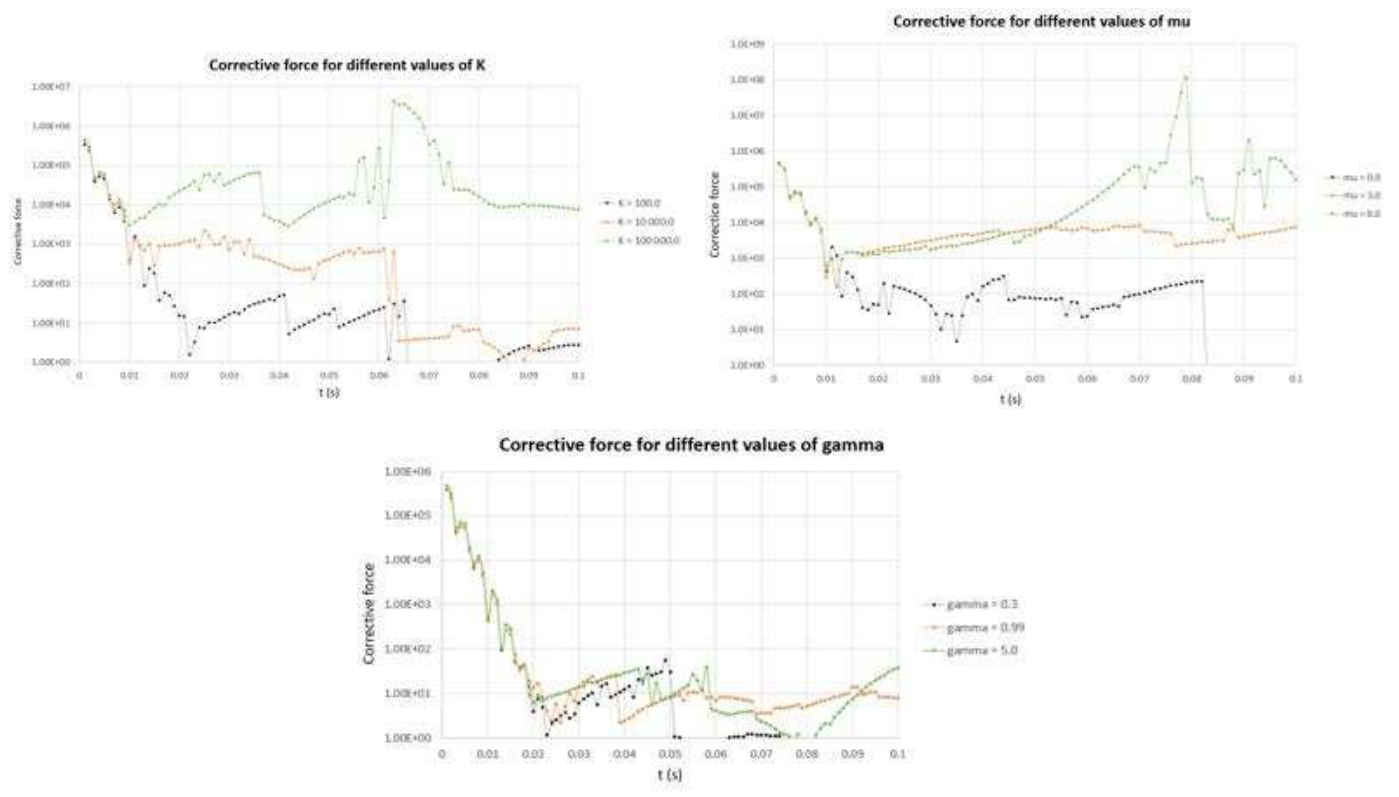

Figure 12: Variation of the corrective force with $\mathrm{K}, \mu$ and $\gamma$

Another way to test the ABM result is evaluating the corrective force that has been implemented to mantain the imposed position and velocity. In this case, Figure 12 reveal, in general, an increase in the 
value of the force necessary to correct the position of the chosen cell when the main parameter of each force rises. As it has been mentioned before, the forces are directly proportional to their main parameters, so they grow with them. Consequently, as the bonding forces between the particles increase, the extraforce that the ABM cell must assume in order to reach the required position will be greater. In other words, when $\mu, \gamma$ or $K$ rise, the union of the particles is higher (they are immersed in a more viscous and cohesive fluid, and also their mechanic union is getting rigid). Because of that union, the effort that must be made by the cell that wants to abandon the position imposed by these forces is greater. This phenomenon is shown in Figure 12, validating the ABM data implementation.

\section{CONCLUSIONS}

A discrete model that could capture the tumoral cells behaviour and tissue properties has been created in a SPH framework. The cohesive and viscous forces have been implemented in order to reproduce the principal characteristics of the tissue where the cells are immersed in. Also, in order to control the behaviour of the cells, a mechanical force has been implemented. Besides, the pressure forces and other parameters as density and mass have been taken into account in the model, leading to a more realistic result. The response of the cells when varying these parameters is the expected one, getting a rigid movement when rising $K$ and a viscous one when rising $\mu$ or $\gamma$.

In addition, an algorithm which opens up the possibility to join the present mechanical model to an $\mathrm{ABM}$ has been implemented. In this study, the input data of this algorithm is imposed, but, with no many changes, the data coming from an ABM could be implemented as its input. Also, the energy consumption, which is related to the extra-force that the cell would do to go from the position where the forces have carried out to the imposed one, is calculated. The proposed strategy to consider these biological data as a force is validated, getting closer to a bigger model which totally reproduces the real growth and evolution of the cells.

Another strength of this model is that lots of study cases would be modelled, thanks to its formulation. The results presented in this study are $2 \mathrm{D}$, but the formulation implemented is prepared to be from $1 \mathrm{D}$ to $3 \mathrm{D}$. By decreasing the time between time steps and increasing the number of study particles, computational and numerical errors would be reduced.

\section{REFERENCES}

[1] M. B. Liu and G. R. Liu Smoothed Particle Hydrodynamics (SPH): an Overview and Recent Developments. Archives of Computational Methods in Engineering (2010).

[2] R. A. Gingold and J. J. Monaghan Smoothed particle hydrodynamics: theory and application to non-spherical stars Monthly Notices of the Royal Astronomical Society (1977)

[3] J. J. Monaghan Smoothed particle hydrodynamics. Annual Review of Astronomy and Astrophysics (1992)

[4] J. J. Monaghan Simulating Free Surface Flows with SPH. Journal of Computational Physics(1994)

[5] Marco Sutti SPH treatment of boundaries and application to moving objects (2019) 